\title{
ARTÍCULO ORIGINAL \\ Entrenamiento de la memoria de trabajo en la enfermedad vascular cerebral: revisión sistemática
}

Fecha de recibido:

9 de septiembre de 2020 .

Fecha de aprobación:

15 de diciembre de 2020 .
Forma de citar este artículo: Landínez DA, Montoya DA. Entrenamiento de la memoria de trabajo en la enfermedad vascular cerebral: revisión sistemática. Med UPB. 2021:40(2):22-32. DOI:10.18566/medupb.v40n2.a04

1 Doctorado en Psicología, Universidad San Buenaventura. Medellín, Colombia

2 Programa de Medicina, Universidad de Manizales. Manizales, Colombia

3 Programa de Psicología, Universidad Católica Luis Amigó. Medellín, Colombia.

${ }^{4}$ Programa de Psicología, Universidad Pontificia Bolivariana. Medellín, Colombia.

Dirección de correspondencia: Daniel Alfredo Landínez Martínez. Correo electrónico: daniel. landinezma@amigo.edu.co

\section{Working Memory Training after cerebrovascular disease: a systematic review / Treinamento Da Memória Operacional Na Doença Cerebrovascular: Revisão Sistemática}

Daniel Alfredo Landínez Martínez ${ }^{1,2,3}$, David Andrés Montoya Arenas ${ }^{1,4}$

\section{RESUMEN}

Objetivo: mejorar la independencia funcional de los pacientes con enfermedad vascular cerebral (EVC) es uno de los objetivos que se plantean los equipos de rehabilitación física y cognitiva. Uno de los modelos que podría dar respuesta a este objetivo es el entrenamiento cognitivo de la memoria de trabajo (MT). Aunque esta estrategia se viene estudiando desde hace 20 años, aún es necesario realizar un estudio de revisión sistemática que permita conocer con claridad los efectos del entrenamiento cognitivo computarizado de la MT en el funcionamiento de la vida diaria en pacientes con EVC isquémica.

Metodología: para ello, se creó una ecuación de búsqueda para aplicar en la base de datos Web of Science (WoS), en el rango temporal desde enero de 2010 a enero de 2019. Posteriormente, se realiza un análisis bibliométrico con el objetivo de identificar los autores y revistas más importantes sobre el tema de investigación. Finalmente, el reporte cronológico identifica la evolución y avances del impacto de los entrenamientos de la memoria de trabajo en la EVC.

Resultados: se encontraron tres enfoques relacionados con: efectos de un programa de realidad virtual en las actividades de la vida diaria, efectos del entrenamiento de la MT en la plasticidad cerebral y efectos del entrenamiento cognitivo de la MT en las actividades de la vida diaria.

Conclusión: se ha propuesto el desempeño cognitivo como uno de los componentes más importantes del funcionamiento en la vida diaria, particularmente la MT como mediador entre los programas de entrenamiento cognitivo y las habilidades funcionales. Los entrenamientos cognitivos reducen el deterioro cognitivo y el riesgo de demencia. Sin embargo, los beneficios de un entrenamiento cognitivo se limitan a dominios muy similares a los entrenados (transferencia cercana, más que lejana).

Palabras clave: enfermedad vascular cerebral; memoria de trabajo; actividades básicas de la vida diaria; entrenamiento cognitivo; rehabilitación

\section{ABSTRACT}

Objective: improving functional independence of patients with cerebrovascular disease has become one of the main goals for physical and cognitive rehabilitation teams. A well-known framework that addresses this issue is Working Memory Training. Although this model has been studied for more than twenty years, a systematic review on the effects of Working Memory Training after ischemic stroke and its impact on everyday functioning is still needed.

Methodology: thus, a search equation has been proposed on Web of Science database from January 2010 to January 2019. Then, a bibliometric analysis presents the most 
important authors and journals in the research topic. Finally, a chronological report shows advanced research lines and effects of Working Memory Training in patients with cerebrovascular disease.

Results: these findings showed 3 approaches related to the following: effects of virtual reality-based programs on everyday functioning, effects of Working Memory Training on brain plasticity, and effects of Working Memory Training on everyday life.

Conclusion: cognitive performance has been proposed to be one of the most important components of functioning in daily life. Working Memory has been shown to strongly mediate between cognitive training and functional abilities. Cognitive training is associated with less cognitive decline and reduced risk of dementia. However, the benefits from cognitive training may be limited to highly similar domains (close transfer rather than far transfer).

Keywords: working memory; stroke; instrumental activities of daily living, cognitive training; rehabilitation

\section{RESUMO}

Introdução: melhorar a independência funcional do paciente com doença cerebrovascular (DCV) é um dos objetivos traçados pelas equipes de reabilitação física e cognitiva. Um dos modelos que poderia responder a esse objetivo é o treinamento cognitivo da memória de trabalho (MT).

Objetivo: embora essa estratégia seja estudada há 20 anos, ainda é necessário realizar um estudo de revisão sistemática que nos permita conhecer claramente os efeitos do treinamento cognitivo computadorizado da MT no funcionamento da vida diária em pacientes com DCV isquêmica.

Metodologia: para isso, foi criada uma equação de busca a ser aplicada na base de dados Web of Science (WoS), no período de janeiro de 2010 a janeiro de 2019. Posteriormente, é realizada uma análise bibliométrica a fim de identificar os autores mais importantes e periódicos sobre o tema de pesquisa. Por fim, o relatório cronológico identifica a evolução e a evolução do impacto do treino da memória de trabalho no CVS. Resultados: foram encontradas três abordagens relacionadas a: efeitos de um programa de realidade virtual nas atividades da vida diária, efeitos do treinamento da MT na plasticidade cerebral e efeitos do treinamento cognitivo da MT nas atividades da vida diária. Conclusão: o desempenho cognitivo tem sido proposto como um dos componentes mais importantes do funcionamento na vida diária, particularmente a MT como mediador entre programas de treinamento cognitivo e habilidades funcionais. $O$ treinamento cognitivo reduz o declínio cognitivo e o risco de demência. No entanto, os benefícios do treinamento cognitivo são limitados a domínios muito semelhantes aos treinados (transferência próxima, em vez de distante).

Palavras-chave: doença vascular cerebral; memória de trabalho; atividades básicas da vida diária; treinamento cognitivo; reabilitação

\section{INTRODUCCIÓN}

Las alteraciones en la MT son comunes en la enfermedad vascular cerebral $(\mathrm{EVC})^{1}$. Por ejemplo, en un estudio se encontró que la prevalencia de la alteración de la memoria de trabajo (MT) era del $49 \%$ entre los participantes con EVC y del 34\% entre pacientes que presentaron buena recuperación clínica ${ }^{2}$. Por otra parte, los pacientes con alteraciones de la MT tienen menor grado de recuperación, con bajo desempeño en actividades básicas e instrumentales de la vida diaria ${ }^{1}$, menores tasas de reincorporación al trabajo, menos participación social, institucionalización y costos más altos en salud ${ }^{3}$. En cuanto al estudio de las actividades instrumentales de la vida diaria (AIVD), aunque no es el eje de investigaciones a largo plazo, varios trabajos con seguimiento a 5-10 años reportan consecuencias negativas de la EVC 4,5. Por ejemplo, las AIVD están afectadas en gran parte de la población con EVC. De un total de 196 pacientes, el $52 \%$ de la muestra reportó que necesitaba ayuda en el cuidado personal y en tareas de la casa ${ }^{6}$. Así mismo, otro estudio describió que después de seis años, solo el 35\% 
de la población se consideraba tan activa como antes de sufrir la $\mathrm{EVC}^{7}$.

En este sentido, los últimos 20 años han sido testigos de múltiples estrategias de rehabilitación en el paciente con EVC como, por ejemplo: el entrenamiento cognitivo de la MT. Ciertos estudios reportan resultados positivos de esta estrategia en términos de ganancias específicas en las tareas entrenadas ${ }^{8}$. También se han identificado efectos del entrenamiento sobre tareas no entrenadas (efectos de transferencia) ${ }^{9}$. Algunos han encontrado efectos significativos de transferencia, mientras que otros no han encontrado ninguno ${ }^{10}$. El entrenamiento de la MT también ha sido pensado para mejorar las AIVD. Sin embargo, algunos aspectos relacionados con la independencia funcional han sido poco evaluados. Dado que la MT es fundamental para el desempeño de AIVD en pacientes con EVC, debe estar muy presente en los estudios de investigación.

En este sentido, el objetivo de un estudio fue investigar si el entrenamiento cognitivo computarizado mejora el funcionamiento cognitivo subjetivo y la calidad de vida después del inicio de la EVC ${ }^{11}$. Un grupo de adultos (30-80 años), con evolución de la EVC entre tres meses a cinco años, fue asignado aleatoriamente a varios subgrupos, uno de intervención $(\mathrm{n}=38)$, otro de control activo (entrenamiento simulado, $\mathrm{n}=35$ ), y uno de lista de espera $(n=24)$. Las mediciones subjetivas fueron el funcionamiento cognitivo (Cuestionario de Falla Cognitiva), el funcionamiento ejecutivo (Cuestionario de Funcionamiento Disejecutivo), la calidad de vida (Encuesta de Salud), las AIVD (Lawton \& Brody) y la vida social (Escala de Participación en Contextos Sociales). Todos los grupos mejoraron en las dos escalas del funcionamiento cognitivo subjetivo y en el funcionamiento ejecutivo subjetivo, pero no en las otras pruebas. Los resultados se mantuvieron cuatro semanas después de completar el entrenamiento. El desempeño del grupo de intervención no fue mejor que el de los otros. Esto sugiere que la mejora se debió a efectos inespecíficos del entrenamiento. Finalmente, se concluyó que el entrenamiento cognitivo computarizado no mejoró el funcionamiento cognitivo subjetivo ni la calidad de vida.

Debido a la inconsistencia de los hallazgos de los estudios, el objetivo de esta revisión sistemática es construir un marco teórico sobre los efectos del entrenamiento cognitivo de MT, en pacientes con EVC isquémica y sobre su impacto en las AIVD.

\section{METODOLOGÍA}

Para la construcción del artículo, se utilizaron herramientas que permitieran un rastreo sobre estudios acerca del entrenamiento de la MT en la EVC. Una de ellas fue la base de datos de la Universidad Nacional de Colombia, en su sistema de red de bibliotecas Sinab. En ella se seleccionó la base de datos WoS para identificar los artículos que describen las palabras clave: working memory training, stroke, e instrumental activities of daily living, con el operador booleano AND. Esta ecuación de búsqueda permitió realizar un análisis bibliométrico entre enero de 2010 y enero de 2019. Este método identifica autores y revistas más relevantes en el tema de investigación ${ }^{12}$. La búsqueda arrojó un total de 77 estudios que cumplieron con los criterios mencionados; ningún artículo fue retirado manualmente, para mejorar la precisión de los resultados analizados utilizando la teoría de grafos.

Una vez obtenidos estos resultados, se realizó un análisis cronológico de la información por medio de la plataforma árbol de la ciencia (web ToS $)^{13}$. ToS posibilita la construcción y entendimiento práctico del marco teórico o estado del arte a partir de la búsqueda inicial en WoS. El algoritmo de ToS se basa en la teoría de grafos, donde los artículos son representados como nodos y las citaciones entre ellos como enlaces. Cada nodo simboliza una unidad de conocimiento ubicada dentro de la red. Los nodos más importantes son identificados a partir de su posición, determinada según los enlaces que conectan a otros nodos ${ }^{14}$. Por tanto, los artículos en la raíz son las referencias seminales de los efectos del entrenamiento cognitivo computarizado de la MT en AIVD, de pacientes con EVC isquémica, los del tronco son los artículos estructurales. Por último, las hojas son artículos que determinan las perspectivas actuales (Figura 1). De esta manera se puede visualizar la información científica en forma de árbol.

\section{RESULTADOS}

En la Tabla 1 (pág. 26) se describen los trabajos más importantes sobre MT y AIVD (ver también la Figura 1).

\section{Artículos de la raíz (seminales)}

Los artículos seminales son trabajos que presentaron una idea de gran importancia dentro de una disciplina. A continuación, se describirán un grupo de estudios que han trabajado sobre la relación entre EVC, AIVD y entrenamiento de la MT (ver Figura 1).

El objetivo de uno de estos estudios fue presentar recomendaciones basadas en la evidencia para la rehabilitación cognitiva, estas sugerencias son producto de una revisión de la literatura sobre la efectividad de la rehabilitación cognitiva para personas con trauma craneoencefálico (TCE) y EVC ${ }^{15}$. Se evaluaron 171 estudios, 29 de Clase I, 35 de Clase II y 107 de Clase III. Finalmente, se resumió la evidencia según el dominio cognitivo de intervención y se realizaron recomendaciones para la práctica clínica 
Figura 1. Principales autores según la ecuación de búsqueda (EVC, AIVD y entrenamiento de la MT).

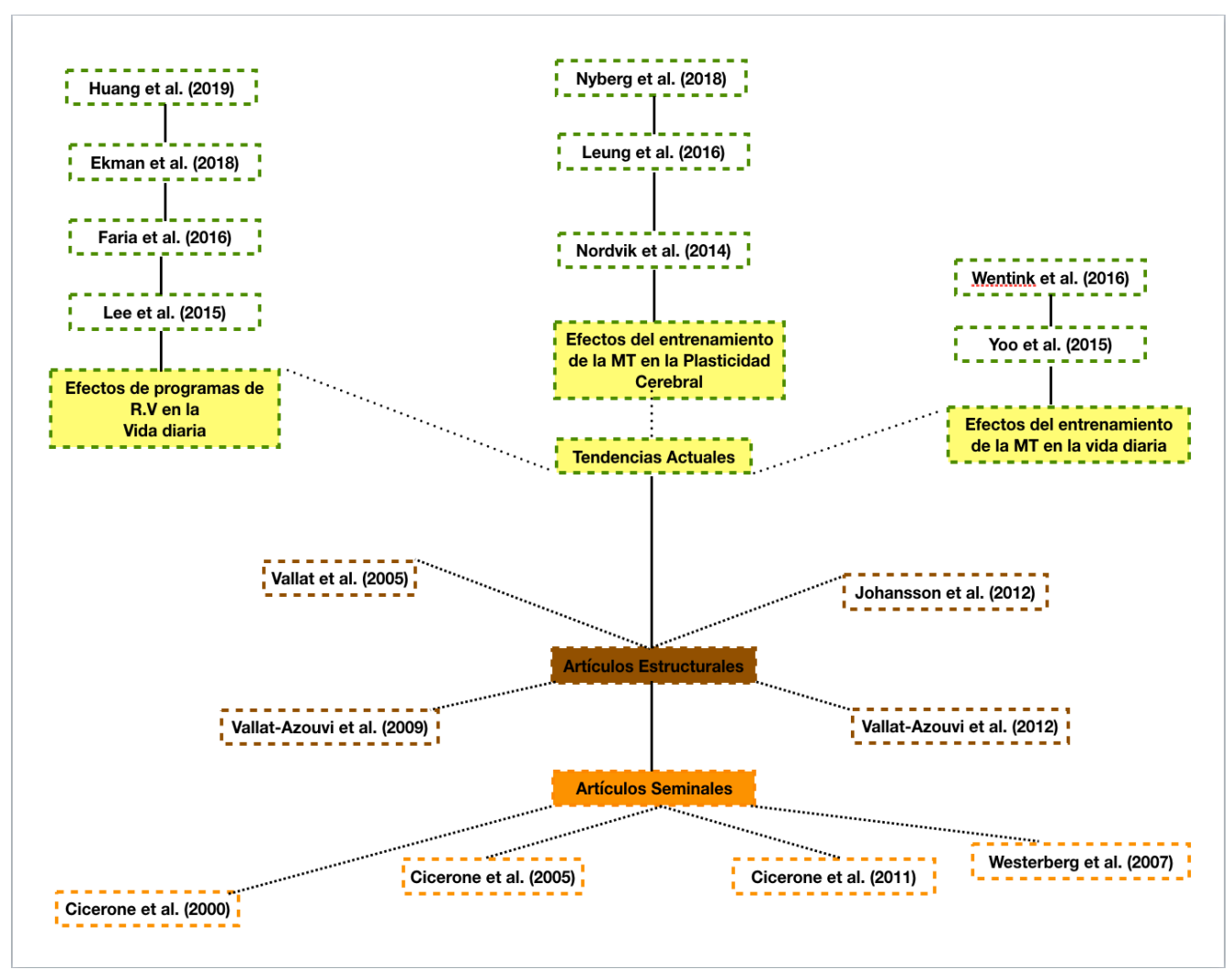

Con respecto a los estudios de intervención de la memoria, cuatro estudios prospectivos controlados (Clase I), con un total de 91 sujetos, evaluaron la efectividad de un entrenamiento cognitivo en sujetos con $\mathrm{EVC}^{16-19}$. Estos estudios compararon la rehabilitación de la memoria con un tratamiento alterno y otros dos incluyeron grupos controles pasivos

Tres de estos estudios ${ }^{16,18,19}$ evidenciaron efectos significativos de la rehabilitación de la MT en los índices neuropsicológicos del funcionamiento de la memoria o disminución en los reportes subjetivos de fallas en la memoria cotidiana. En el cuarto estudio ${ }^{17}$, los beneficios de la recuperación de la memoria fueron evidentes cuando los sujetos se estratificaron de acuerdo con la gravedad de su deterioro inicial de la memoria. La evidencia también sugiere que la recuperación de la memoria es más efectiva cuando los sujetos son independientes para las AIVD y cuando están motivados para continuar con el uso de estrategias de rehabilitación. Los autores concluyen que se deben generar intervenciones dirigidas a facilitar la adquisición de habilidades específicas, en lugar de mejorar el funcionamiento de la memoria en $s^{20}$.

Siguiendo la misma línea, cinco años más tarde se publicó una actualización del estudio de revisión que cubrió el periodo entre 1998-2002 ${ }^{21}$. De los 87 estudios evaluados, 17 fueron clasificados como Clase I, 8 de Clase II, y 62 de Clase III. Se resumió la evidencia dentro de cada área de intervención y se realizaron recomendaciones para la práctica clínica y guías para la generación de políticas de salud pública.

Los estudios relacionados con la rehabilitación de la memoria continúan utilizando estrategias compensatorias. También ha crecido el interés en evaluar el uso de los entrenamientos cognitivos computarizados. Dos estudios Clase I, con 41 sujetos, demostraron la efectividad de estrategias mnemotécnicas para pacientes con alteraciones de la memoria y EVC ${ }^{22-23}$. Estos hallazgos son consistentes con cuatro estudios previos Clase I que respaldan el uso de estrategias mnemotécnicas ${ }^{15}$. Estas intervenciones consisten en estrategias internas (imágenes visuales), así como en técnicas de compensación mediante diarios y cuadernos de notas. El principal beneficio de estas técnicas es sobre la memoria verbal ${ }^{22}$. La incorporación de técnicas de autogestión mejora el uso de un cuaderno de notas para la memoria ${ }^{23}$. Así pues, estas intervenciones facilitan la finalización de las AIVD que los sujetos identifican como relevantes.

Con base en la evidencia actual y en conjunto con los resultados del artículo de revisión previo, se recomienda el uso de entrenamientos cognitivos computarizados 


\section{Aspectos metodológicos}

Muestra: 171 artículos (Clase I: 29, Clase II: 35, Clase III: 107).

Objetivo: describir recomendaciones basadas en la evidencia sobre los

efectos de la rehabilitación cognitiva en pacientes con EVC.

\section{Muestra: 87 artículos (Clase I: 17, Clase II: 8, Clase III: 62)}

Objetivo: actualizar las recomendaciones basadas en la evidencia sobre los efectos de la rehabilitación cognitiva en pacientes con EVC entre 1998-2002.

Muestra: 112 artículos (Clase I: 19, Clase II: 11, Clase III: 82).

Objetivo: actualizar recomendaciones basadas en la evidencia sobre los efectos de la rehabilitación cognitiva en pacientes con EVC entre 2003-2008.

\section{Muestra: 18 participantes con EVC}

Objetivo: evaluar los efectos de un entrenamiento de MT en pacientes con EVC.

\section{Muestra: estudio de Caso con EVC izquierda.}

Objetivo: evaluar el efecto de una terapia de MT en un paciente con EVC con alteraciones en tareas que dependían del funcionamiento adecuado de ejecutivo central y el bucle fonológico.

\section{Muestra: (2) estudios de caso con EVC izquierdo.}

Objetivo: evaluar el efecto de un programa de rehabilitación en tareas dependientes del funcionamiento adecuado del ejecutivo central en pacientes con lesión cerebral.

\section{Muestra: 451 participantes con EVC.}

Objetivo: desarrollar una escala para medir las consecuencias de las alteraciones de la MT en tareas de la vida diaria.

\section{Muestra: 18 pacientes con EVC.}

Objetivo: evaluar si pacientes con lesión cerebral se podrían beneficiar de un entrenamiento de MT y si el software impacta las actividades de la vida diaria y la salud psicológica.

Muestra: 10 pacientes con EVC.

Objetivo: determinar los efectos de un programa de realidad virtual utilizando el sistema de ejercicios y rehabilitación interactiva (SERI) en la recuperación de la función motor, cognitiva y en el desempeño de las actividades de la vida diaria.
País

Resultados

Fuente

EE. UU. La evidencia sugiere que la recuperación de la memoria es más efectiva cuando Cicerone et al. los sujetos son independientes en AIVD, y cuando están motivados para continuar (2000). con el uso de estrategias de rehabilitación.

Se deben generar intervenciones para facilitar la adquisición de habilidades específicas en lugar de mejorar el funcionamiento de la memoria per se.

EE. UU. Se recomienda el uso de entrenamientos cognitivos computarizados con Cicerone et al. aplicación directa a AIVD como una guía de práctica en sujetos con deterioro (2005). moderado o grave de la memoria después de EVC.

Investigaciones a futuro deben ir más allá de la pregunta de si la rehabilitación es efectiva, y evaluar factores específicos de la intervención y características de los pacientes que permitan optimizar los beneficios de la rehabilitación cognitiva.

EE. UU. El aprendizaje sin errores se puede utilizar para enseñar estrategias Cicerone etal. compensatorias a la hora de ejecutar tareas de memoria que involucran AIVD, (2011). como por ejemplo tomarse la medicina en un horario específico y guardar las llaves en un mismo lugar.

EE. UU. Se encontró reducción significativa de los síntomas de fallas cognitivas a la hora Westerberg et de realizar AIVD. Este tipo de intervenciones se debe realizar durante la fase al. (2007). crónica y no en las etapas tempranas del EVC.

Francia Se encontró un mejor desempeño en AIVD que requieren de la MT como: ir de Vallat et al. compras, interacción social, una conversación por teléfono. Hubo aumento del desempeño en tareas como el span de dígitos (directo e inverso).

Francia Se reporta aumento del desempeño en tareas diseñadas para el ejecutivo central, Vallat et al. de la misma manera, se encontró que las puntuaciones mejoraron en tareas de (2009). aritmética y resolución de problemas.

Francia El cuestionario tiene buena consistencia interna tanto en sujetos con lesión Vallat et al. cerebral (Alfa de Cronbach 0.89 y 0.94, respectivamente). En sujetos sanos, se (2012). encontraron efectos significativos de la edad ( $p$ 0.0001) y del nivel de escolaridad (p 0.01).

Suecia Los autores reportan que en el cuestionario de fallos cognitivos se encontró Johansson et una reducción de las alteraciones cognitivas experimentadas por los pacientes. al. (2012). También se reportó una disminución de síntomas depresivos y de ansiedad.

Korea Este estudio encontró mejorías significativas en la capacidad para ejecutar Lee et al. actividades de la vida diaria evidenciado en las puntuaciones del índice de (2015). Barthel, así como en el funcionamiento de miembros superiores. 


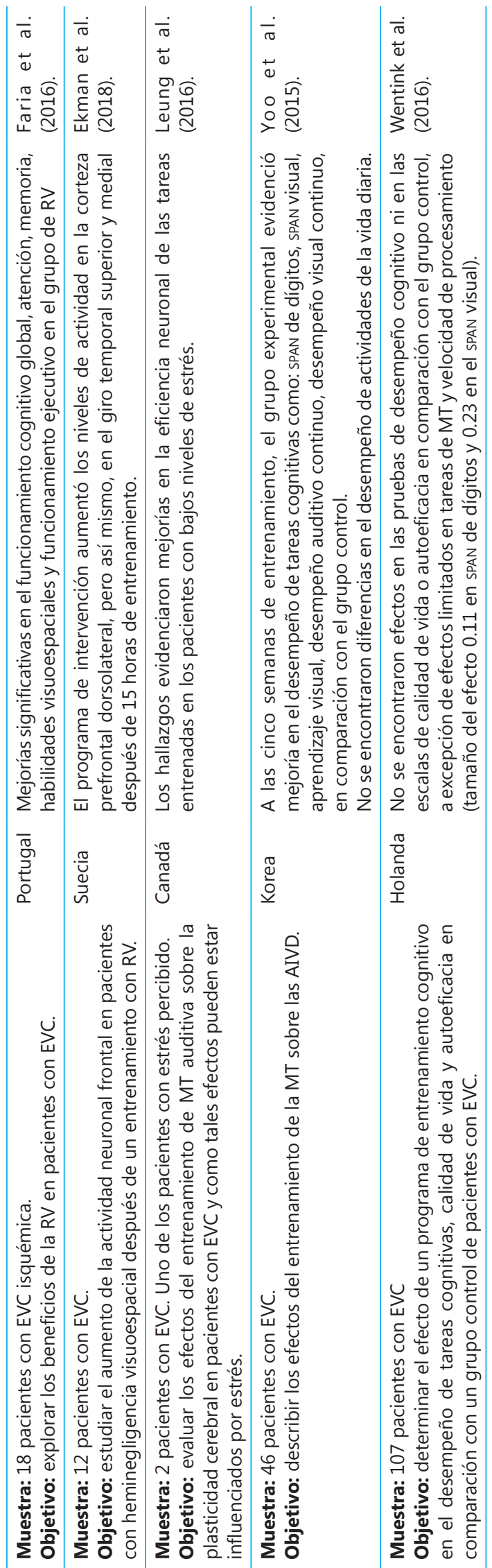

con aplicación directa sobre las AIVD, como una guía de práctica en sujetos con deterioro moderado o grave de la memoria después de $\mathrm{EVC}^{15}$. Las investigaciones a futuro deben ir más allá de la pregunta de si la rehabilitación es efectiva, y evaluar factores específicos de la intervención y las características de los pacientes que permiten optimizar el pronóstico.

Después de este artículo de revisión, se desarrolló un proyecto de investigación que, en atención a las sugerencias con respecto al uso de entrenamientos computarizados, evaluó los efectos de un entrenamiento de MT en pacientes con $\mathrm{EVC}^{24}$. El grupo experimental y el de control fueron aleatorizados a recibir entrenamiento computarizado de MT o seguimiento pasivo. El entrenamiento se desarrolló durante cinco semanas. Los autores hallaron que el grupo experimental mejoró en las pruebas que miden MT y atención. Además, hubo reducción de los fallos cognitivos al realizar AIVD. Los investigadores concluyen que este tipo de intervenciones se debe realizar durante la fase crónica y no en las etapas tempranas de la EVC, ya que en la fase crónica los pacientes intentan recuperar sus hábitos de trabajo y son conscientes de sus déficits, por lo que se pueden motivar más para entrenar funciones cognitivas perdidas ${ }^{25}$.

Para terminar con los artículos seminales, se presenta un metanálisis del mismo equipo ${ }^{15,21,26}$. Al igual que en los trabajos previos, el objetivo fue realizar recomendaciones basadas en la evidencia para la rehabilitación cognitiva en pacientes con EVC en el periodo correspondiente a 2003-2008.

Respecto a los déficits de memoria, se revisaron un estudio Clase $\mathrm{I}^{27}$, uno Clase $\mathrm{II}^{28}$ y cuatro Clase $\mathrm{III}^{29-32}$, que investigaron los beneficios del aprendizaje sin errores en la rehabilitación de la memoria.

El estudio Clase $\mathrm{I}^{27}$ comparó un entrenamiento computarizado de memoria con una terapia asistida por un profesional en pacientes con EVC, las dos intervenciones utilizaron el aprendizaje sin errores (20 sesiones de entrenamiento de memoria, entrenamiento en AIVD y consolidación y generalización de habilidades). E1 resultado fue siempre un mejor desempeño en pruebas neuropsicológicas de memoria.

En el estudio Clase II $^{28}$ se evaluó el efecto de un programa de instrucciones en pacientes con EVC con alteraciones de memoria. El entrenamiento consistía en enseñar cómo enviar un correo electrónico utilizando la estrategia de aprendizaje sin errores y una técnica metacognitiva. Hubo una fuerte relación entre el programa de instrucciones y la tarea de enviar el correo electrónico, donde el buen desempeño se mantuvo durante un mes. Sin embargo, no se demostró transferencia a una tarea nueva con exigencias diferentes. Este estudio concluye que el aprendizaje sin errores se puede utilizar para enseñar estrategias compensatorias para tareas de memoria que 
involucran AIVD, como tomarse la medicación en un horario específico y guardar las llaves en un mismo lugar ${ }^{30}$.

\section{Artículos del tronco (estructurales)}

Son los que le comienzan a dar forma a la relación entre EVC, AIVD y entrenamiento cognitivo de la MT (Figura 1).

El objetivo de uno de estos estudios fue evaluar el efecto de una terapia de MT en un paciente con EVC y alteración en tareas que dependían del funcionamiento del ejecutivo central y el bucle fonológico ${ }^{33}$. La rehabilitación incluía el entrenamiento del almacenamiento y procesamiento de la MT verbal. El resultado del entrenamiento fue medido con tareas de MT: el span de dígitos, span visuoespacial, efecto de similitud fonológica, efecto de longitud de la palabra, paradigma Brown-Peterson, tareas complejas relacionadas y dos cuestionarios ecológicos para medir efectos en AIVD. Hubo mejoría en tareas como el span de dígitos (directo e inverso) y en la tarea Brown-Peterson, así como en áreas que no fueron entrenadas (aritmética). Aún más importante, mejoró el desempeño en las AIVD que requieren MT, como hacer compras, interacción social o conversación por teléfono. Los autores concluyen que los resultados no se deben a la recuperación espontánea, en la medida en que el desempeño cognitivo de los pacientes era constante antes de la terapia.

Otro trabajo evaluó un programa de rehabilitación en tareas dependientes del ejecutivo central en pacientes con lesión cerebral ${ }^{34}$. La MT se revisó mediante el span de dígitos (directo e inverso), el span visuoespacial, el paradigma Brown Peterson y la tarea n-back. También se utilizaron tareas inespecíficas que requerían MT (aritmética y resolución de problemas) y un cuestionario para evaluar actividades de la vida diaria. El entrenamiento constaba de doce tareas de dificultad ascendente que involucraban capacidad y grado de procesamiento. La capacidad estaba relacionada con la cantidad de información (número de palabras), el grado estaba relacionado con la complejidad del estímulo (frecuencia de uso). Los estímulos se presentaron uno por segundo. El entrenamiento se realizaba dos veces por semana durante ocho meses. Hubo mejoría en tareas relacionadas con el ejecutivo central, la aritmética y la resolución de problemas y se evidenció reducción en las fallas atencionales durante las actividades de la vida diaria.

Las actividades de la vida diaria son una dimensión que ha cobrado interés en los estudios sobre entrenamiento de la MT. De hecho, se desarrolló una escala para medir las consecuencias de las alteraciones de la MT sobre las actividades de la vida diaria ${ }^{35}$. El cuestionario de MT (30 preguntas) es una escala autoadministrada de tres dimensiones: almacenamiento a corto plazo, atención y control ejecutivo. En una muestra de 313 participantes sanos y 69 con EVC, este cuestionario mostró buena consistencia interna en ambos tipos de participantes (alfa de Cronbach 0.89 y 0.94 , respectivamente). El cuestionario de MT tiene la sensibilidad adecuada para discriminar pacientes de controles en los tres dominios y buena validez concurrente con el cuestionario de fallos cognitivos. Así mismo, la puntuación total en las quejas cognitivas se correlacionó con otras medidas de MT (span visuoespacial y memoria a corto plazo) y con la capacidad intelectual (matrices de Raven).

El desarrollo de escalas para medir el impacto de los déficits en MT en las actividades de la vida diaria ha permitido que los estudios sobre entrenamiento de la MT cobren mayor interés en la literatura. Ejemplo de lo anterior es una investigación que evaluó si pacientes con lesión cerebral se benefician del entrenamiento de la MT y si hay mejoría en las actividades de la vida diaria y en la salud mental ${ }^{36}$. Este estudio utilizó un programa de entrenamiento computarizado (Cogmed $\mathrm{QM})$ en 18 pacientes con EVC, entre los 17-64 años, que tenían dificultades en actividades de la vida diaria relacionadas con la MT. Se halló una reducción de las alteraciones cognitivas, así como la disminución de síntomas depresivos y de ansiedad. Un hallazgo fundamental es la correlación entre puntuaciones bajas al comenzar el estudio y un buen desempeño en el entrenamiento. Es decir, los participantes con menor capacidad de MT fueron los más beneficiados.

\section{Artículos de las hojas (tendencias actuales)}

Los trabajos descritos sentaron las bases de las perspectivas actuales. Estas investigaciones tratan subtemas específicos (tendencia) sobre los efectos del entrenamiento cognitivo computarizado de la MT en las AIVD en pacientes con EVC isquémica. La presente revisión sistemática evidenció tres tendencias: i) efectos de un programa de realidad virtual en las actividades de la vida diaria, ii) efectos del entrenamiento de la MT en la plasticidad cerebral y iii) efectos del entrenamiento cognitivo de la MT en las actividades de la vida diaria (Figura 1).

\section{Efectos de un programa de realidad virtual en las actividades de la vida diaria}

Entre las estrategias más novedosas en la rehabilitación de pacientes con EVC se encuentra la rehabilitación asistida por robots o realidad virtual $(\mathrm{RV})^{37}$. En esta línea, un estudio determinó los efectos de un programa de RV basado en ejercicios y rehabilitación interactiva (SERI) sobre la recuperación motora y cognitiva sobre el desempeño de las actividades de la vida diaria en 10 pacientes con $\mathrm{EVC}^{38}$. Los pacientes debían entrenarse 
con el programa de RV durante 30 minutos cada día, tres veces a la semana, por cuatro semanas. La función cognitiva mejoró significativamente después de cuatro semanas. De la misma manera, se observaron mejorías en las actividades de la vida diaria y en el funcionamiento motor de los miembros superiores. El estudio concluye que la rehabilitación mediante RV es efectiva, aunque sugiere que deben desarrollarse protocolos de entrenamiento que permitan una ejercitación más estructurada.

En un ensayo controlado aleatorizado en 18 pacientes de dos unidades de rehabilitación, nueve individuos se entrenaron por medio de RV y los demás recibieron rehabilitación convencional. La RV involucraba simulación de una ciudad (Reh@City), en donde las tareas de memoria, atención, habilidades visuoespaciales y funciones ejecutivas ejercitaban rutinas de la vida diaria. Los autores reportan mejorías significativas después de la intervención en el funcionamiento cognitivo global, la atención, la memoria, las habilidades visuoespaciales y el funcionamiento ejecutivo en el grupo de RV. El grupo control mejoró al seguimiento en la velocidad de procesamiento y en la atención, así como en la memoria y participación social. El grupo de RV tuvo mejor desempeño cognitivo global, atención, y funcionamiento ejecutivo en comparación con el que recibió terapia convencional.

Una investigación reciente estudió la actividad neuronal frontal en pacientes con heminegligencia visuoespacial, después de un entrenamiento con RV (RehAtt) ${ }^{40}$, el cual combinó estimulación visual, auditiva y sensoriomotor. La intervención se desarrolló tres veces a la semana, durante cinco semanas. Los efectos del entrenamiento en la actividad cerebral se evaluaron mediante resonancia magnética funcional. La intervención aumentó la actividad en la corteza prefrontal dorsolateral y en el giro temporal superior y medial. Dado que los efectos más significativos se observaron en las regiones prefrontales, relacionadas con conductas dirigidas a una meta y adaptativas y con el mantenimiento de categorías, los cambios ocurrieron específicamente en el campo ocular frontal y en la corteza frontal ventral.

La información disponible se resume en que la RV en pacientes con EVC es efectiva, aunque es necesario crear protocolos estructurados de entrenamiento y estudiar qué actividades de la vida diaria mejoran más ${ }^{41}$.

\section{Efectos del entrenamiento de la MT en la plasticidad cerebral}

Los avances en neuroimagen han modificado de dos maneras el conocimiento sobre las alteraciones cognitivas y físicas después de una lesión cerebral ${ }^{42}$ : i) la resonancia magnética funcional permite monitorear los procesos de plasticidad cerebral inducidos por la rehabilitación, mientras el paciente está ejecutando la intervención y ii) el mapeo cerebral permite relacionar estructuras y redes neuronales con funciones cerebrales específicas. Lo anterior posibilita explicar la relación entre lesiones y síntomas, especialmente en áreas difíciles de evaluar con pruebas tradicionales.

Esto se lleva a la práctica en estudios que han demostrado que el volumen de la sustancia gris aumenta en respuesta al aprendizaje intensivo de una habilidad nueva ${ }^{43}$. De la misma manera, los cambios en la sustancia gris ocurren después de aprender estrategias mnemotécnicas ${ }^{44}$, técnicas de estudio ${ }^{45}$, y práctica de tareas espaciales computarizadas ${ }^{46}$. También, se ha encontrado actividad en la sustancia blanca después del entrenamiento de la $\mathrm{MT}^{47,48}$. Un estudio encontró cambios en sustancia blanca, producto de un entrenamiento cognitivo computarizado en un paciente con $\mathrm{EVC}^{49}$.

Siguiendo esta misma línea de trabajo, otro estudio evaluó los efectos del entrenamiento de la MT auditiva sobre la plasticidad cerebral en pacientes con EVC y cómo dichos efectos son influenciados por estrés ${ }^{50}$. Los pacientes con alto grado de estrés tuvieron mejores puntuaciones en las escalas de satisfacción del desempeño. Por otra parte, los pacientes con bajos niveles de estrés percibido presentaron mejor desempeño en todas las valoraciones. La resonancia magnética evidenció mejorías en la eficiencia neuronal de las tareas entrenadas, en los pacientes con poco estrés. Estos resultados indican que es necesario tener en cuenta variables psicológicas como el estrés, cuando se estudia la plasticidad cerebral en pacientes con EVC.

\section{Efectos del entrenamiento de la MT sobre las actividades de la vida diaria}

Otra línea de trabajo sobre el entrenamiento cognitivo focaliza en los efectos sobre las actividades de la vida diaria. Un estudio sobre 46 pacientes aleatorizados en dos grupos, donde el grupo experimental recibió rehabilitación y entrenamiento computarizado (The RehaaCom) durante 30 minutos, cinco días a la semana, por cinco semanas, y el de control recibió solo terapia de rehabilitación que incluía terapia ocupacional y física, reporta que en el grupo experimental mejoró el desempeño de varias tareas cognitivas (sPAN de dígitos, SPAN visual, aprendizaje visual, desempeño auditivo continuo, desempeño visual continuo). Sin embargo, no hubo gran mejoría en el desempeño de actividades de la vida diaria. Los autores sugieren que el tiempo de evolución de la EVC, el área lesionada y el desempeño cognitivo previo podrían tener que ver con sus hallazgos ${ }^{51}$.

Un estudio similar se propuso determinar el efecto de un entrenamiento sobre tareas cognitivas, calidad de vida y autoeficacia en pacientes con $\mathrm{EVC}^{52}$. Los pacientes, que tenían percepción de mal desempeño 
cognitivo, fueron asignados aleatoriamente a un grupo experimental y a uno de control. La intervención fue el programa denominado Lumosity Inc. El grupo control recibió información general acerca del cerebro. No hubo diferencia entre los grupos en el desempeño cognitivo, ni en las escalas de calidad de vida o autoeficacia, a excepción de un efecto limitado del entrenamiento sobre la MT y la velocidad de procesamiento. Estos hallazgos indican que la intervención no es efectiva, aunque queda por estudiar si modular el grado de intensidad en los entrenamientos influye sobre la evolución de los pacientes ${ }^{53}$.

\section{DISCUSIÓN}

En esta revisión sistemática se presentan tres perspectivas que dominan el estudio de los efectos del entrenamiento cognitivo computarizado de la MT en las AIVD, en pacientes con $\mathrm{EVC}$ isquémica, desde hace aproximadamente 20 años. A pesar de identificar el gran interés por los programas de RV y sus efectos en la plasticidad cerebral, el estudio del impacto sobre las AIVD aún es insipiente. Los trabajos en esta línea se centran en observar el desempeño en tareas de MT, sin datos concluyentes sobre el desempeño en el diario vivir de los pacientes con EVC.

Aun así, los entrenamientos computarizados de la MT tienen un potencial importante, ya que permiten generar situaciones que tengan que ver con las actividades de la vida diaria (habilidades funcionales), como la actividad física, intelectual y laboral. En estos entrenamientos la MT actúa como moderador entre las tareas cognitivas y las habilidades funcionales, ya que en la vida diaria se requiere almacenamiento activo, manipulación de información e integración de señales, procesos centrales en la MT.

Como consecuencia de estos antecedentes, los autores están probando la iniciativa BRAINING en un ensayo, para determinar el efecto del trabajo computarizado en la MT sobre la cognición y las AIVD en pacientes con EVC isquémico (ensayo: RIDIE-STUDY-ID5e61da9608f23). BRAINING es un software de seis actividades desarrolladas bajo los principios del modelo de MT de Baddeley. Es decir, tienen como fundamento la capacidad de almacenar y manipular información verbal y espacial. Por ejemplo, en una actividad llamada rotación se presenta una serie de cubos que se iluminan aleatoriamente. El software comenzará con una secuencia de dos cubos, el intervalo entre estímulos es de dos segundos. Luego, los cubos rotarán 90 grados. El paciente debe recordar el orden y posición en que aparecieron los cubos que se iluminaron antes. El ensayo tiene en cuenta reportes previos sobre la duración e intensidad de cada sesión y el grado de dificultad de cada actividad. Como consecuencias de la contingencia sanitaria por la pandemia del virus SARS-CoV-2, se está probando el funcionamiento de BRAINING mediante telepsicología.

En conclusión, la MT es uno de los componentes más importantes del funcionamiento. La MT media entre los programas de entrenamiento cognitivo y las habilidades funcionales. Sin embargo, la evidencia disponible señala que los beneficios de un entrenamiento cognitivo se limitan a dominios muy similares a los entrenados.

\section{Fuentes}

Este artículo es producto de la investigación: Efectos del entrenamiento cognitivo computarizado de la memoria de trabajo en la enfermedad vascular cerebral, inscrito en la Vicerrectoría de Investigaciones de la Universidad San Buenaventura.

\section{Agradecimientos}

Un gran agradecimiento a la Universidad Católica Luis Amigó y al doctor Rigoberto González Piña por su apoyo para el diseño, producción y ejecución del software de entrenamiento computarizado de memoria de trabajo, BRAINING.

\section{Declaración de conflictos de intereses}

El autor principal es el creador y propietario de la iniciativa BRAINING (http://www.strokeapp.org).

\section{REFERENCIAS}

1. Leśniak M, Bak T, Czepiel W, Seniów J, Członkowska A. Frequency and prognostic value of cognitive disorders in stroke patients. Dement Geriatr Cogn Disord 2008;26:356-63.

2. Jokinen H, Melkas S, Ylikoski R, Pohjasvaara T, Kaste M, Erkinjuntti T, et al. Post-stroke cognitive impairment is common even after successful clinical recovery. Eur J Neurol 2015;22:1288-94.

3. Ownsworth T, Shum D. Relationship between executive functions and productivity outcomes following stroke. Disabil Rehabil 2008;30:531-40.

4. Wolfe CDA, Crichton SL, Heuschmann PU, McKevitt CJ, Toschke AM, Grieve AP, et al. Estimates of outcomes up to ten years after stroke: Analysis from the Prospective South London Stroke Register. Plos Med 2011;8:e1001033. 
5. Feigin VL, Barker-Collo S, Parag V, Senior H, Lawes CMM, Ratnasabapathy $\mathrm{Y}$, et al. Auckland stroke outcomes study: Part 1: Gender, stroke types, ethnicity, and functional outcomes 5 years poststroke. Neurology 2010;75:1597-607.

6. Walsh ME, Galvin R, Loughnane C, Macey C, Horgan NF. Community re-integration and long-term need in the first five years after stroke: Results from a national survey. Disability and Rehabilitation 2015;37:1834-8

7. Singam A, Ytterberg C, Tham K, Von Koch L. Participation in complex and social everyday activities six years after stroke: Predictors for return to pre-stroke level. Plos One 2015;10:e0144344.

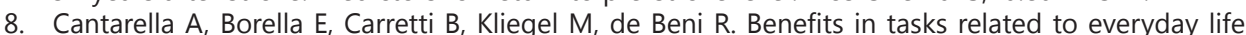
competences after a working memory training in older adults. Int J Geriatr Psychiatry 2017;32:86-93.

9. Karbach J, Verhaeghen P. Making working memory work: A meta-analysis of executive-control and working memory training in older adults. Psychol Sci 2014;25:2027-37.

10. Borella E, Carretti B, Cantarella A, Riboldi F, Zavagnin M, De Beni R. Benefits of training visuospatial working memory in young-old and old-old. Dev Psychol 2014;50:714-27.

11. Van De Ven RM, Murre JMJ, Buitenweg JIV, Veltman DJ, Aaronson JA, Nijboer TCW, et al. The influence of computer-based cognitive flexibility training on subjective cognitive well-being after stroke: A multi-center randomized controlled trial. Plos One 2017;12:e0187582.

12. Vallaster C, Kraus S, Merigó Lindahl JM, Nielsen A. Ethics and entrepreneurship: A bibliometric study and literature review. J Bus Res 2019;99:226-37.

13. Robledo S, Osorio GAG, López C. Networking en pequeña empresa: una revisión bibliográfica utilizando la teoria de grafos. Rev Vínculos 2014;11:6-16.

14. Hirsch JE. An index to quantify an individual's scientific research output. Pnas 2005;102;16569-72.

15. Cicerone KD, Dahlberg C, Kalmar K, Langenbahn DM, Malec JF, Bergquist TF, et al. Evidence-based cognitive rehabilitation: Recommendations for clinical practice. Arch Phys Med Rehabil 2000;81:1596-615.

16. Berg IJ, Koning-Haanstra M, Deelman BG. Long-term effects of memory rehabilitation: A controlled study. Neuropsychol Rehabil 1991;2;97-111.

17. Ryan T V., Ruff RM. The efficacy of structured memory retraining in a group comparison of head trauma patients. Arch Clin Neuropsychol 1988;3:165-79.

18. J KM, Mary A. Computer delivery of memory retraining with head injured patients. In: Cognitive Rehabilitation 1985;3;26-31.

19. Schmitter-Edgecombe $M$, Fahy JF, Whelan JP, Long CJ. Memory remediation after severe closed head injury: Notebook training versus supportive therapy. J Consult Clin Psychol 1995;63:484-9.

20. Kirsch NL, Levine SP, Lajiness-O'neill R, Schnyder M. Computer-assisted interactive task guidance: Facilitating the performance of a simulated vocational task. J Head Trauma Rehabil 1992;7;13-25.

21. Cicerone KD, Dahlberg C, Malec JF, Langenbahn DM, Felicetti T, Kneipp S, et al. Evidence-based cognitive rehabilitation: updated review of the literature from 1998 through 2002. Arch Phys Med Rehabil 2005:86:1681-92.

22. Kaschel R, Della Sala S, Cantagallo A, Fahlböck A, Laaksonen R, Kazen M. Imagery mnemonics for the rehabilitation of memory: A randomised group controlled trial. Neuropsychol Rehabil 2002;12:127-153.

23. Ownsworth TL, McFarland K. Memory remediation in long-term acquired brain injury: Two approaches in diary training. Brain Inj 1999;13:605-26.

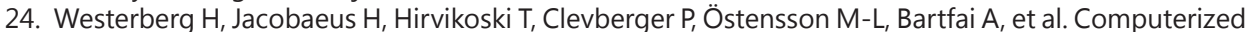
working memory training after stroke-A pilot study. Brain Inj 2007;21:21-9.

25. Stambrook M, Moore AD, Peters LC, Deviaene C, Hawryluk GA. Effects of mild, moderate and severe closed head injury on long-term vocational status. Brain Inj 1990;4:183-90.

26. Cicerone KD, Langenbahn DM, Braden C, Malec JF, Kalmar K, Fraas M, et al. Evidence-based cognitive rehabilitation: Updated review of the literature from 2003 through 2008. Archives of Physical Medicine and Rehabilitation 2011;92:519-30.

27. Dou ZL, Man DWK, Ou HN, Zheng JL, Tam SF. Computerized errorless learning-based memory rehabilitation for Chinese patients with brain injury: A preliminary quasi-experimental clinical design study. Brain Inj 2006;20:219-25.

28. Ehlhardt LA, Sohlberg MM, Glang A, Albin R. TEACH-M: A pilot study evaluating an instructural sequence for persons with impaired memory and excutive functions. Brain Inj 2005;19:569-83.

29. Campbell L, Wilson FC, McCann J, Kernahan G, Rogers RG. Single case experimental design study of Carer facilitated Errorless Learning in a patient with severe memory impairment following TBI. NeuroRehabilitation 2007;22:325-33.

30. Melton AK, Bourgeois MS. Training compensatory memory strategies via the telephone for persons with TBI. Aphasiology 2005;19:353-64.

31. Pitel AL, Beaunieux $H$, Lebaron $N$, Joyeux $F$, Desgranges $B$, Eustache $F$. Two case studies in the application of errorless learning techniques in memory impaired patients with additional executive deficits. Brain Inj 2006;20:1099-110.

32. Tam SF, Man WK. Evaluating computer-assisted memory retraining programmes for people with post-head injury amnesia. Brain Inj 2004;18:461-70.

33. Vallat $C$, Azouvi $P$, Hardisson $H$, Meffert $R$, Tessier $C$, Pradat-Diehl P. Rehabilitation of verbal working memory after left hemisphere stroke. Brain Inj 2005;19:1157-64.

34. Vallat-Azouvi C, Pradat-Diehl P, Azouvi P. Rehabilitation of the central executive of working memory after severe traumatic brain injury: Two single-case studies. Brain Inj 2009;23:585-94. 
35. Vallat-Azouvi C, Pradat-Diehl P, Azouvi P. The Working Memory Questionnaire: A scale to assess everyday life problems related to deficits of working memory in brain injured patients. Neuropsychol Rehabil. 2012;22:634-49.

36. Johansson B, Tornmalm M. Working memory training for patients with acquired brain injury: Effects in daily life. Scand J Occup Ther 2012;19:176-83.

37. Cho K, Yu J, Jung J. Effects of virtual reality-based rehabilitation on upper extremity function and visual perception in stroke patients: A randomized control trial. J Phys Ther Sci 2013;24:1205-8.

38. Lee K-H. Effects of a virtual reality-based exercise program on functional recovery in stroke patients: part 1. J Phys Ther Sci 2015;27:1637-40.

39. Faria AL, Andrade A, Soares L, I Badia SB. Benefits of virtual reality based cognitive rehabilitation through simulated activities of daily living: A randomized controlled trial with stroke patients. J Neuroeng Rehabil 2016;13:96.

40. Ekman U, Fordell H, Eriksson J, Lenfeldt N, Wåhlin A, Eklund A, et al. Increase of frontal neuronal activity in chronic neglect after training in virtual reality. Acta Neurol Scand 2018;138:284-92.

41. Huang Q, Wu W, Chen X, Wu B, Wu L, Huang X, et al. Evaluating the effect and mechanism of upper limb motor function recovery induced by immersive virtual-reality-based rehabilitation for subacute stroke subjects: Study protocol for a randomized controlled trial. Trials 2019;20:104.

42. Nordvik JE, Walle KM, Nyberg CK, Fjell AM, Walhovd KB, Westlye LT, et al. Bridging the gap between clinical neuroscience and cognitive rehabilitation: The role of cognitive training, models of neuroplasticity and advanced neuroimaging in future brain injury rehabilitation. NeuroRehabilitation 2014;34:81-5.

43. Driemeyer J, Boyke J, Gaser C, Büchel C, May A. Changes in gray matter induced by learning Revisited. Plos One 2008;3:e2669.

44. Engvig A, Fjell AM, Westlye LT, Moberget T, Sundseth O, Larsen VA, et al. Effects of memory training on cortical thickness in the elderly. Neuroimage 2010;52:1667-76.

45. Draganski B, Gaser C, Kempermann G, Kuhn HG, Winkler J, Buchel C, et al. Temporal and spatial dynamics of brain structure changes during extensive learning. J Neurosci 2006;26:6314-7.

46. Haier RJ, Karama S, Leyba L, Jung RE. MRI assessment of cortical thickness and functional activity changes in adolescent girls following three months of practice on a visual-spatial task. BMC Res Notes 2009;2:174.

47. Fields RD. White matter in learning, cognition and psychiatric disorders. Trends in Neurosciences 2008;31:361-70.

48. Takeuchi H, Sekiguchi A, Taki Y, Yokoyama S, Yomogida Y, Komuro N, et al. Training of working memory impacts structural connectivity. J Neurosci 2010;30:3297-303.

49. Nordvik JE, Schanke AK, Walhovd K, Fjell A, Grydeland H, Landrø NI. Exploring the relationship between white matter microstructure and working memory functioning following stroke: $A$ single case study of computerized cognitive training. Neurocase 2012;18:139-51.

50. Leung AWS, Barrett LM, Butterworth D, Werther K, Dawson DR, Brintnell ES. Neural plastic effects of working memory training influenced by self-perceived stress in stroke: A case illustration. Front Psychol 2016;7:1266.

51. Yoo C, Yong M, Chung J, Yang Y. Effect of computerized cognitive rehabilitation program on cognitive function and activities of living in stroke patients. J Phys Ther Sci 2015;27:2487-9.

52. Wentink MM, Berger MAM, de Kloet AJ, Meesters J, Band GPH, Wolterbeek R, et al. The effects of an 8-week computer-based brain training programme on cognitive functioning, QoL and self-efficacy after stroke. Neuropsychol Rehabil. 2016;26:847-65.

53. Guye S, Röcke C, Martin M, von Bastian CC. Functional ability in everyday life: Are associations with an engaged lifestyle mediated by working memory? Journals Gerontol Ser B 2019;11;1-11. 